\section{Cárie dentária em população ribeirinha do Estado de Rondônia, Região Amazônica, Brasil, $2005 / 2006$}

\author{
Dental caries in a riverine community in Rondônia \\ State, Amazon Region, Brazil, 2005-2006
}

\begin{abstract}
This study aimed to analyze dental caries patterns among riverine people from Rondônia State, Brazil (Machado and Preto rivers) in 2005 and 2006. A total of 469 subjects were examined, using the World Health Organization form, under natural light, using a wooden tongue depressor and CPI probe in cases of doubts about the presence of dental caries. The results were: 4-5-year age bracket, dmtf $=4.30$ and $19.64 \%$ caries-free; 6 -10 years, DMTF $=1.04, \mathrm{dmtf}=3.52$ and $17.05 \%$ caries-free; 12 years, DMTF $=2.65$ and $30.76 \%$ caries-free; 18 years, DMTF $=5.41$ and $19.51 \%$ caries-free; $35-44$ years, DMTF = 17.74 and $2.98 \%$ caries-free; $65-74$ years, DMTF $=21.56$ and $4.34 \%$ caries-free. When each component was analyzed separately in the dmtf and DMTF indices, decay was most prevalent in the 0-3, 4-5, 6-10, 12, and 18-year brackets. However, in young and older adults, the most prevalent component was missing teeth. In conclusion, the study population showed a high dental caries index, thus highlighting the need for educational, preventive, and curative measures.
\end{abstract}

Dental Caries; Oral Health; Oral Diagnosis

\author{
Ricardo Henrique Alves da Silva 1 \\ Roberta Francisca Martins de Castro 2 \\ Diana Carla Soares Cunha 2 \\ Carina Thais de Almeida 3 \\ José Roberto de Magalhães Bastos 3 \\ Luis Marcelo Aranha Camargo 4
}

\section{Introdução}

As condições de saúde bucal em nível individual e coletivo são avaliadas, principalmente, pela análise da ocorrência de cárie dentária. O declínio da prevalência da cárie, em populações jovens, pode ser notado mundialmente, principalmente nas últimas três décadas, nas mais diferentes localidades 1,2,3.

Em populações social e economicamente desfavorecidas, a prevalência de doenças passíveis de prevenção geralmente se apresenta mais elevada quando comparada com localidades mais desenvolvidas. A cárie dentária também segue esse padrão de distribuição, seja por falta de informação da população sobre os cuidados com a higiene bucal ou por falta de acesso ao tratamento odontológico 4,5,6,7,8,9.

Quanto à região do estudo, observa-se que o inquérito SB Brasil 200310 constatou os seguintes índices para a Região Norte: ceod 1,34 (até os 3 anos de idade); ceod 3,22 (aos 5 anos de idade); CPOD 3,13 (aos 12 anos de idade); CPOD 6,14 (15-19 anos de idade); CPOD 19,88 (35-44 anos de idade); CPOD 28,34 (65-74 anos de idade).

E inserida nessa região, a população do $\mathrm{Mu}$ nicípio de Porto Velho, Estado de Rondônia, local do presente estudo, caracteriza-se por possuir 371.791 habitantes, sendo cerca de $30 \%$ residente em área rural e rural-ribeirinha 11.

Dessa forma, o objetivo deste trabalho é apresentar os dados do levantamento de cárie dentá- 
ria realizado na população residente às margens dos rios Machado e Preto, a fim de possibilitar uma análise das condições de saúde bucal da população.

\section{Material e métodos}

Inicialmente, o projeto de levantamento epidemiológico foi aprovado pelo Comitê de Ética em Pesquisa da Faculdade de Odontologia de Bauru, da Universidade de São Paulo, através do processo no. 82/2004, seguindo as normas da Resolução $n^{o}$. 196/96 do Conselho Nacional de Saúde.

A população-alvo do estudo foi composta pela população residente às margens dos rios Machado e Preto, região norte do Estado de Rondônia, sendo ambos afluentes do rio Madeira.

Estima-se que a população estudada conte com cerca de 800 habitantes, baseado em estimativas da Prefeitura Municipal de Porto Velho, não possuindo nenhum tipo de serviço de saúde e apresenta, como dificuldade, o fato de a viagem feita por barco demorar cerca de vinte horas até Porto Velho, sendo que a maioria da população não possui condições financeiras para custear tal viagem.

As coletas dos dados foram realizadas durante três viagens, nos anos de 2005 (fevereiro e julho) e 2006 (janeiro), sendo a população do estudo selecionada por conveniência, ou seja, moradores presentes nas localidades durante os períodos de coleta que autorizaram a realização do exame odontológico.

O exame clínico foi realizado com luz natural, nas dependências do Barco-hospital Floriano Riva Filho (pertencente ao Município de Porto Velho e Ministério da Saúde) e nas visitas domiciliares, realizadas por dois examinadores previamente calibrados, utilizando espátulas de madeira descartáveis, espelhos clínicos planos $n^{\circ} .5$ e equipamento de proteção individual. Não se utilizou sonda exploradora, sendo a sonda CPI utilizada quando havia dúvidas da presença de uma lesão cariosa, em virtude do grande número de exames e dificuldades na esterilização.

Toda a população que foi até o Barco-hospital recebeu atendimento clínico (nas áreas de odontologia, medicina, nutrição, farmácia e enfermagem, além de exames laboratoriais), independentemente da participação no levantamento epidemiológico.

O procedimento de calibração foi realizado através do exame de duas seqüências de vinte indivíduos cada, apresentando as mais variadas condições de cárie dentária, a fim de contemplar todas as condições dos critérios diagnósticos. Os exames foram realizados no período da manhã, e os reexames, no período da tarde, em voluntários, no Centro de Saúde Bucal Prof. Dr. João Adolfo Caldas Navarro, em Monte Negro, Rondônia, onde está localizada a sede do Instituto de Ciências Biomédicas 5 (ICB-5), dois dias antes da primeira viagem. A concordância verificada pelo teste kappa $(\mathrm{k}$ ) indicou $\mathrm{k}=0,85$ (interexaminadores), $\mathrm{k}=0,92 \mathrm{e} \mathrm{k}=0,90$ (intraexaminador, respectivamente examinador 1 e 2 ), consideradas satisfatórias.

Durante o levantamento epidemiológico, realizaram-se exames em duplicata em $5 \%$ da amostra, nos três primeiros e três últimos dias de exame de cada viagem, a fim de observar se a concordância era mantida em um nível satisfatório. A concordância verificada pelo teste kappa indicou $\mathrm{k}=0,86$ (interexaminadores), $\mathrm{k}=0,94 \mathrm{e}$ $\mathrm{k}=0,93$ (intraexaminador, respectivamente examinador 1 e 2).

O modelo de ficha utilizado foi o estabelecido pela Organização Mundial da Saúde (OMS) 12, utilizando os critérios estabelecidos para as condições dentárias, tanto para dentes decíduos, como para permanentes: cariado, restaurado (com cárie e sem cárie), extraído, hígido e excluído.

Optou-se, neste estudo, pela análise estatística descritiva dos índices CPOD e ceod, seguido do desvio-padrão (DP), assim como o percentual de livres de cárie e a proporção por componente do CPOD, tabulado por meio do Microsoft Office Excel 2003 (Microsoft Corp., Estados Unidos).

\section{Resultados}

O tamanho da amostra de estudo, dividido por faixas etárias, ficou com a distribuição exposta na Tabela 1, perfazendo 469 sujeitos da pesquisa.

Os resultados obtidos nolevantamento epidemiológico demonstram o índice de cárie (CPOD

Tabela 1

Distribuição da amostra de estudo, por faixa etária, 2005/2006.

\begin{tabular}{lc}
\hline Faixa etária (anos) & Amostra \\
\hline $0-3$ & 104 \\
$4-5$ & 55 \\
$6-10$ & 128 \\
12 & 51 \\
18 & 41 \\
$35-44$ & 67 \\
$65-74$ & 23 \\
Total & 469 \\
\hline
\end{tabular}


e/ou ceod, de acordo com a faixa etária), seguido pela medida do DP, bem como o percentual de livres de cárie, os quais podem ser observados na Tabela 2 .

O número de indivíduos livres de cárie indicou: na faixa etária de 0-3 anos de idade (n = 69; 66,35\%); de 4-5 anos ( $\mathrm{n}=11 ; 19,64 \%)$; de $6-10$ anos ( $\mathrm{n}=22$; $17,05 \%)$; aos 12 anos $(\mathrm{n}=16$; $30,76 \%)$; aos 18 anos ( $\mathrm{n}=8 ; 19,51 \%)$; de $35-44$ anos ( $\mathrm{n}=2 ; 2,98 \%)$; de $65-74$ anos $(\mathrm{n}=1 ; 4,34 \%)$, (Tabela 2).

$\mathrm{Na}$ análise de cada um dos componentes do índice CPOD, observa-se que o componente cariado tem maior relevância nas idades de 0-3, 45, 6-10, 12 e 18 anos. Já nas faixas etárias adulta (35-44) e idosa (65-74), o componente que mais contribui com o valor do índice é o componente perdido (Tabela 3).

E, na Tabela 4, está representado o percentual de indivíduos, de acordo com os componentes dos índices de cárie dentária, dividido por faixa etária, demonstrando a grande contribuição do componente cariado nas faixas 0-3, 4-5, 6-10 e
12 anos de idades, dos componentes cariado e perdido, nas faixas etárias de 18 e 35-44 anos, e do componente perdido na faixa 65-74 anos de idade.

\section{Discussão}

Do ponto de vista epidemiológico, a cárie é, possivelmente, a doença bucal de maior relevância. Sua estreita associação com dieta e estilo de vida também lhe confere relevância antropológica 13. Na população estudada, pôde ser observado que a dieta, baseada principalmente em produtos de pesca, caça, plantações e criações locais, devido ao distanciamento e isolamento de centros urbanos, aliada a um grande desconhecimento sobre a saúde bucal e práticas insuficientes em higiene oral conduzem ao quadro epidemiológico observado.

A amostragem por conveniência realizada neste estudo pode gerar potenciais vieses de seleção, pois não há como saber se todas as pessoas

Tabela 2

Índices CPOD e ceod, desvio-padrão (DP) e livres de cárie, por faixa etária, na população rural ribeirinha, rio Machado e rio Preto, Estado de Rondônia, Brasil, 2005/2006.

\begin{tabular}{lccc}
\hline Faixa etária (anos) & CPOD (DP) & ceod (DP) & Livres de cárie (\%) \\
\hline $0-3$ & - & $1,50(2,68)$ & 66,35 \\
$4-5$ & - & $4,30(3,48)$ & 19,64 \\
$6-10$ & $1,04(1,50)$ & $3,52(3,44)$ & 17,05 \\
12 & $2,65(3,01)$ & $0,38(1,27)$ & 30,76 \\
18 & $5,41(5,33)$ & - & 19,51 \\
$35-44$ & $17,74(8,61)$ & - & 2,98 \\
$65-74$ & $21,56(11,95)$ & - & 4,34 \\
\hline
\end{tabular}

Tabela 3

Média por componentes dos índices de cárie dentária e desvio-padrão (DP), por faixa etária, na população rural ribeirinha, rio Machado e rio Preto, Estado de Rondônia, Brasil, 2005/2006.

\begin{tabular}{lccc}
\hline Faixa etária (anos) & Componente cariado (DP) & Componente restaurado (DP) & Componente perdido (DP) \\
\hline $0-3$ & $1,47(2,68)$ & $0,02(0,20)$ & $0,01(0,09)$ \\
$4-5$ & $4,20(3,44)$ & $0,11(0,49)$ & $0,00(0,00)$ \\
$6-10$ & $4,26(4,83)$ & $0,23(0,90)$ & $0,07(0,31)$ \\
12 & $2,15(4,03)$ & $0,21(0,89)$ & $0,29(0,72)$ \\
18 & $3,17(3,69)$ & $0,71(1,75)$ & $1,54(2,89)$ \\
$35-44$ & $2,54(3,66)$ & $0,86(2,99)$ & $14,33(8,83)$ \\
$65-74$ & $1,96(3,13)$ & $0,04(0,20)$ & $19,56(11,92)$ \\
\hline
\end{tabular}


Percentual de indivíduos, por componentes dos índices de cárie dentária, por faixa etária, na população rural ribeirinha, rio Machado e rio Preto, Estado de Rondônia, Brasil, 2005/2006.

\begin{tabular}{|c|c|c|c|c|c|c|}
\hline \multirow[t]{2}{*}{ Faixa etária (anos) } & \multicolumn{2}{|c|}{ Componente cariado } & \multicolumn{2}{|c|}{ Componente restaurado } & \multicolumn{2}{|c|}{ Componente perdido } \\
\hline & $\operatorname{ceod}(\%)$ & CPOD (\%) & $\operatorname{ceod}(\%)$ & CPOD (\%) & ceod $(\%)$ & CPOD (\%) \\
\hline $0-3$ & 34,00 & - & 0,96 & - & 0,96 & - \\
\hline $4-5$ & 81,82 & 3,64 & 5,45 & - & - & \\
\hline $6-10$ & 67,19 & 39,06 & 7,03 & 7,81 & - & 5,47 \\
\hline 12 & 13,72 & 58,82 & 3,92 & 11,76 & - & 19,61 \\
\hline 18 & - & 73,17 & - & 21,95 & - & 53,66 \\
\hline $35-44$ & - & 64,18 & - & 20,89 & - & 95,52 \\
\hline $65-74$ & - & 43,48 & - & 8,69 & - & 91,30 \\
\hline
\end{tabular}

incluídas na amostra são representativas da população, não possibilitando mensuração do erro amostral e impedindo uma declaração conclusiva sobre os resultados obtidos 14,15. Porém, pelo desconhecimento e dificuldades de localização da população estudada, adotou-se esse modelo de amostragem.

Dentre as faixas etárias, o grupo etário de 6574 anos estudado não alcançou o número mínimo recomendado de quarenta pessoas 12 , atingindo apenas 23 sujeitos da pesquisa, sendo essa limitação importante e devendo ser ressaltada para possíveis vieses na comparação com outros dados populacionais.

Os dados indicam, na população estudada, CPOD $=2,65$ aos 12 anos de idade. Narvai et al. ${ }^{3}$ relatam que o índice CPOD, na Região Norte, declinou de 7,50 (em 1986) para 3,13 (em 2003), aos 12 anos de idade, sendo a média nacional observada, em 1986, CPOD = 6,65 e, em 2003, $\mathrm{CPOD}=2,79$.

Além disso, aos 12 anos de idade, 33\% da população estudada possui CPOD > 3,0; e 30\% são livres de cárie, sugerindo uma concentração da doença cárie, fato observado em diversos estudos, nas mais diferentes populações 3,7,16,17,18. Nesse sentido, Narvai et al. 3 afirmam que, no Brasil, 20\% dos escolares passaram a concentrar $60 \%$ da carga da doença.

A população estudada apresenta, como característica marcante, um isolamento dos centros urbanos devido aos elevados custos para viagem até o Município de Porto Velho, porém, não se trata de população indígena, possuindo hábitos de vida exemplificados pela agricultura de subsistência, caça e pesca, além de hábitos em saúde bucal de origem popular e folclórica 19 .

Sendo assim, no trabalho de Arantes et al. 13, no qual foram examinados 228 índios Xavánte
(Brasil), observam-se CPOD = 3,70 (12-14 anos), $\mathrm{CPOD}=4,60$ (15-19 anos), $\mathrm{CPOD}=15,05$ (30-39 anos) e CPOD = 19,60 (50 anos ou mais), dados semelhantes com a população do presente estudo.

A influência para a configuração de tais índices de cárie dentária refere-se, de acordo com DeAngelis \& Warren 6, à falta de acesso aos cuidados em saúde bucal, consumo de água não fluoretada e a necessidade de educação em saúde bucal, fato esse confirmado por diversos autores 20,21. Em nosso estudo, foram observadas características semelhantes, respaldadas pelo elevado número de atendimentos clínicos realizados em todas as viagens.

Considerando-se os dados coletados, observou-se que o componente cariado esteve mais presente nas idades de 0-3, 4-5, 6-10, 12 e 18 anos. Nas comunidades examinadas, a cobertura assistencial em saúde é precária, não existindo posto de saúde na maioria delas e contando com a escassa presença de profissionais da saúde na maior parte do tempo.

Nas faixas etárias de 35-44 anos e 65-74 anos, observa-se uma maior contribuição por parte do componente perdido, indicando que o problema da cárie dentária só é resolvido quando não há mais condição de se recuperar o elemento dentário, sendo a exodontia a principal estratégia assistencial na localidade, resultado também observado por pesquisadores em regiões com estrutura semelhante 13 .

Além disso, os sistemas de saúde pública foram, historicamente, curativos e excludentes 9,22, especialmente em relação a populações residentes em zona rural 23, levando aos quadros observados, neste estudo, para a população de maior faixa etária. Essa prática assistencial mutiladora, condenada nos dias atuais, continua sendo pra- 
ticada na região, sendo necessária à reabilitação dessa população, por meio de próteses totais e removíveis, possíveis de serem realizadas no Barco-hospital Floriano Riva Filho.

Abreu et al. 23 concluem, em seu trabalho, que há uma tendência de queda no índice CPOD no meio rural brasileiro, mesmo ainda sendo os índices de cárie maiores na população rural que na urbana 24,25 .

E, conforme observado no SB Brasil 2003 10, para a Região Norte, verificou-se que a contribuição por componentes do índice ceod, entre 18-36 meses, foi 1,31 (cariado), 0,02 (obturado) e 0,01 (perdido); e aos cinco anos de idade foi de 2,89 (cariado), 0,19 (obturado) e 0,15 (perdido). Os dados vão de encontro com os achados da presente pesquisa, sendo maiores aos cinco anos na população ribeirinha.

E, ainda no SB Brasil 2003 10, aos 12 anos de idade, o componente cariado responde por 2,27 em um $\mathrm{CPOD}=3,13$. No presente estudo, responde por 2,15 em um CPOD = 2,65.

Já em zona rural do Estado de São Paulo, o mais desenvolvido da nação brasileira, no $\mathrm{Mu}$ nicípio de Itapetininga, verificou-se ceod $=2,63$ (aos cinco anos) e CPOD = 2,45 (aos 12 anos), em escolares da zona rural, sendo os dados de cárie dentária, aos 12 anos, bastante próximos aos da população ribeirinha estudada em Rondônia 26.

$\mathrm{E}$, apesar de serem antigas, quanto às metas da Federation Dentaire Internacionale (FDI) 27 e OMS 28, é possível observar que, para o ano
2000, almejavam-se alguns itens: aos cinco anos de idade, 50\% dos indivíduos livres de cárie; aos 12 anos de idade, CPOD menor e/ou igual a 3,0; aos 18 anos de idade, $80 \%$ dos indivíduos com todos os dentes presentes na boca; na faixa etária de 35-44 anos, 75\% dos indivíduos com vinte ou mais dentes presentes na boca; e na faixa de 6574 anos, 50\% dos indivíduos com vinte ou mais dentes na boca.

No presente estudo, foi observado que apenas uma das cinco metas supracitadas foi atingida, haja vista que: aos cinco anos de idade, apenas dois sujeitos da pesquisa (dentre 19 examinados) apresentaram CPOD $=0$, perfazendo $10,53 \%$; o CPOD, aos 12 anos de idade, foi 2,65; aos 18 anos de idade, 46,34\% apresentaram todos os dentes presentes na boca; na faixa etária de 35-44 anos, apenas $29,85 \%$ dos sujeitos da pesquisa apresentaram vinte ou mais dentes na boca; e, na faixa de 65-74 anos, apenas 17,39\% apresentaram vinte ou mais dentes na boca.

\section{Conclusão}

O índice de cárie dentária apresenta-se elevado, em todas as faixas etárias analisadas, na população residente às margens dos rios Machado e Preto, durante os anos 2005/2006, no Estado de Rondônia, sendo de extrema importância a implementação de programas educativos, preventivos e curativos na região. 


\section{Resumo}

O objetivo foi analisar experiência de cárie dentária na população ribeirinha residente às margens dos rios Machado e Preto (Rondônia, Brasil), em 2005 e 2006. Foram examinados 469 indivíduos com formulário preconizado pela Organização Mundial da Saúde, sob luz natural e utilização de espátulas de madeira e sonda CPI. Na faixa etária de 4-5 anos de idade, ceod = 4,30 e 19,64\% livres de cárie; 6-10 anos, $C P O D=1,04$, ceod $=3,52,17,05 \%$ livres de cárie; aos 12 anos, $C P O D=2,65$ e 30,76\% livres de cárie; aos 18 anos, $C P O D=5,41$ e 19,51\% livres de cárie; 35 44 anos, $C P O D=17,74$ e 2,98\% livres de cárie; $65-74$ anos, $C P O D=21,56$ e 4,34\% livres de cárie. Na análise por componentes, constatou-se que o componente cariado tem maior prevalência nas idades de 0-3, 4-5, 6-10, 12 e 18 anos. Em adultos e idosos, o componente que mais contribui é o perdido. Conclui-se que a população apresenta índices de cárie dentária elevados sendo necessária a atuação em âmbito educativo, preventivo e curativo.

Cárie Dentária; Saúde Bucal; Diagnóstico Bucal

\section{Referências}

1. Marthaler TM, O'Mullane DM, Vrbic V. The prevalence of dental caries in Europe 1990-1995. ORCA Saturday afternoon symposium 1995. Caries Res 1996; 30:237-55.

2. Nadanovsky P, Sheiham A. Relative contribution of dental services to the changes in caries level of 12year-old children in 18 industrialized countries in the 1970s and early 1980s. Community Dent Oral Epidemiol 1995; 23:331-9.

3. Narvai PC, Frazão P, Roncalli AG, Antunes JLF. Cárie dentária no Brasil: declínio, polarização, iniqüidade e exclusão social. Rev Panam Salud Pública 2006; 19:385-93.

4. Cangussu MCT, Castellanos-Fernandes RA. Prevalência de cárie dentária em escolares de 12 e 15 anos de Salvador, Bahia, 2001. Rev Bras Saúde Matern Infant 2004; 4:287-97.

5. Cortelli SC, Cortelli JR, Prado JS, Aquino DR, Jorge AOC. Fatores de risco à cárie e CPOD em crianças com idade escolar. Ciênc Odontol Bras 2004; 7:7582.

6. DeAngelis S, Warren C. Establishing community partenerships: providing better oral health care to undeserved children. J Dent Hyg 2001; 75:310-5.

\section{Colaboradores}

R. H. A. Silva e R. F. M. Castro participaram da elaboração e execução do projeto de pesquisa e da confecção do artigo científico. D. C. S. Cunha, C. T. Almeida e J. R M. Bastos, colaboraram na execução do projeto de pesquisa e confecção do artigo científico. L. M. A. Camargo contribuiu na elaboração, execução e coordenação do projeto de pesquisa e da confecção do artigo científico.

\section{Agradecimentos}

Ao Conselho Nacional de Desenvolvimento Científico e Tecnológico (CNPq) pelo fomento da pesquisa. À tripulação do Barco-hospital Floriano Riva Filho pela atenção e colaboração, à população ribeirinha pela cooperação e hospitalidade e à prefeitura de Porto Velho, na pessoa do Prefeito Roberto Sobrinho, pelo constante apoio nas etapas da pesquisa.
7. Gushi LL, Rihs LB, Wada RS, Sousa MLR. Cárie dentária em crianças de escolas públicas e privadas do Município de Porto Feliz, São Paulo, Brasil. Arq Odontol 2003; 39:257-63.

8. Milgrom P. Tooth decay in our poorest children: what can we do? J Indiana Dent Assoc 2000-2001; 79:24-6.

9. Nomura LH, Bastos JLD, Peres MA. Dental pain prevalence and association with dental caries and socioeconomic status in schoolchildren, Southern Brazil, 2002. Braz Oral Res 2004; 18:134-40.

10. Ministério da Saúde. Projeto SB Brasil: condições de saúde bucal da população brasileira, 20022003. Resultados principais. Brasília: Ministério da Saúde; 2004.

11. Instituto Brasileiro de Geografia e Estatística. Contagem da população 2007. http://www.ibge.gov. $\mathrm{br} /$ home/estatistica/populacao/contagem2007/ RO.pdf (acessado em 06/Nov/2007).

12. Organização Mundial da Saúde. Levantamento epidemiológico básico de saúde bucal: manual de instruções. Brasília: Organização Mundial da Saúde; 1999. 
13. Arantes R, Santos RV, Coimbra Jr. CEA. Saúde bucal na população indígena Xavánte de Pimentel Barbosa, Mato Grosso, Brasil. Cad Saúde Pública 2001; 17:375-84.

14. Churchill G. Marketing research: methodological foundations. New York: The Dryden Press; 1998.

15. Kinnear TC, Taylor JR. Marketing research: an applied aproach. New York: McGraw-Hill; 1979.

16. MacKeown JM, Cleaton-Jones PE, Fatti P. Caries and micronutrient intake among urban South African children: a cohort study. Community Dent Oral Epidemiol 2003; 31:213-20.

17. Sales-Peres SHC, Bastos JRM. Perfil epidemiológico de cárie dentária em crianças de 12 anos de idade, residentes em cidades fluoretadas e não fluoretadas, na região centro-oeste do Estado de São Paulo. Cad Saúde Pública 2002; 18:1281-8.

18. Weyne SC. A construção do paradigma de promoção de saúde: um desafio para as novas gerações. In: Kriger L, organizador. Promoção de saúde bucal. São Paulo: Editora Artes Médicas; 2003. p. 3-26.

19. Silva RHA, Castro RFM, Caldana ML, Sales-Peres A, Sales-Peres SHC, Bastos JRM. Desafios em promoção de saúde bucal: abordagem antropológicocultural e epidemiológica de população ribeirinha em RO. Pesqui Odontol Bras 2004; 18 Suppl:29.

20. Freire MCM, Batista SMO. Prevalência de cárie e necessidade de tratamento em escolares de seis a doze anos da rede pública de ensino. Rev Saúde Pública 1994; 33:124-30.
21. Lalloo R, Myburgh NG, Hobdell MH. Dental caries, socio-economic development and national oral health policies. Int Dent J 1999; 49:196-202.

22. Abreu MHNG, Werneck MAF. Sistema incremental no Brasil: uma avaliação histórica. Arq Odontol 1998; 34:121-31.

23. Abreu MHNG, Modena CM, Pordeus IA. Populações residentes em zona rural e cárie dentária: revisão sistemática. RFO UPF 2004; 9:48-54.

24. Cangussu MCT, Coelho EO, Castellanos-Fernandes RA. Epidemiologia e iniqüidade em saúde bucal aos 5, 12 e 15 anos de idade no Município de Itatiba, São Paulo, 2000. Rev Fac Odontol Bauru 2001; 9:77-85.

25. Tagliaferro EPS, RihsLB, Sousa MLR. Prevalência de cárie, fluorose dentária e necessidades de tratamento em escolares, Leme, SP. Arq Odontol 2002; 38:213-21.

26. Mello TRC. Planejamento de serviços em saúde bucal para a zona rural [Dissertação de Mestrado]. São Paulo: Faculdade de Odontologia, Universidade de São Paulo; 2002.

27. Federation Dentaire Internacionale. Global goals for oral health in the year 2000. Int Dent J 1982; 32:74-7.

28. World Health Organization. Oral health global indicators for 2.000: DMFT -3 at 12 years. Geneva: World Health Organization; 1985.

Recebido em 27/Ago/2007

Versão final reapresentada em 11/Fev/2008

Aprovado em 25/Fev/2008 